\title{
Cardiac protective effects of irbesartan via the PPAR-gamma signaling pathway in angiotensin- converting enzyme 2-deficient mice
}

Zhen-Zhou Zhang ${ }^{1,2+}{ }^{+}$, Qian-Hui Shang ${ }^{3+}$, Hai-Yan Jin ${ }^{1,4}$, Bei Song ${ }^{1,2}$, Gavin Y Oudit ${ }^{5}$, Lin Lu ${ }^{6}$, Tong Zhou ${ }^{7}$, Ying-Le X ${ }^{1,2}$, Ping-Jin Gao ${ }^{1,2^{*}}$, Ding-Liang Zhu ${ }^{1,2}$, Josef M Penninger ${ }^{8}$ and Jiu-Chang Zhong ${ }^{1,2^{*}}$

\begin{abstract}
Background: Angiotensin-converting enzyme 2 (ACE2), a monocarboxypeptidase which metabolizes angiotensin II (Ang II) to generate Ang-(1-7), has been shown to prevent cardiac hypertrophy and injury but the mechanism remains elusive. Irbesartan has the dual actions of angiotensin receptor blockade and peroxisome proliferatoractivated receptor- $\gamma$ (PPAR $\gamma$ ) activation. We hypothesized that irbesartan would exert its protective effects on ACE2 deficiency-mediated myocardial fibrosis and cardiac injury via the PPARY signaling.

Methods: 10-week-old ACE2 knockout (ACE2KO; Ace2 $\left.{ }^{-1 / y}\right)$ mice received daily with irbesartan $(50 \mathrm{mg} / \mathrm{kg})$ or saline for 2 weeks. The wild-type mice (Ace ${ }^{+/ y}$ ) were used to the normal controls. We examined changes in myocardial ultrastructure, fibrosis-related genes and pathological signaling by real-time PCR gene array, Western blotting, Masson trichrome staining and transmission electron microscope analyses, respectively.

Results: Compared with the Ace $2^{+/ y}$ mice, cardiac expression of PPARa and PPARY were reduced in Ace ${ }^{-/ y}$ mice and the myocardial collagen volume fraction (CVF) and expression of fibrosis-related genes were increased, including transforming growth factor- $\beta 1$ (TGF $\beta 1$ ), connective tissue growth factor (CTGF), collagen I and collagen III. Moreover, ACE2 deficiency triggered cardiac hypertrophy, increased myocardial fibrosis and adverse ultrastructure injury in ACE2KO hearts with higher levels of atrial natriuretic factor (ANF) and phosphorylated extracellular signalregulated kinase 1/2 (ERK1/2), without affecting cardiac systolic function. Intriguingly, treatment with irbesartan significantly reversed ACE2 deficiency-mediated pathological hypertrophy and myocardial fibrosis in Ace $2^{-1 y}$ mice linked with enhancement of plasma Ang-(1-7) level and downregulation of AT1 receptor in heart. Consistent with attenuation of myocardial fibrosis and ultrastructure injury, the myocardial CVF and levels of ANF, TGF $\beta 1$, CTGF, collagen I, collagen III and phosphorylated ERK1/2 were lower, and expression of PPARY was higher in ACE2KO mice in response to irbesartan treatment, without affecting cardiac expression of PPARa, PPAR $\delta$, $\beta$-myosin heavy chain, TGF 32 and fibronectin.

(Continued on next page)
\end{abstract}

\footnotetext{
* Correspondence: gaopingjin@sibs.ac.cn; jiuchangzhong@aliyun.com

${ }^{\dagger}$ Equal contributors

'State Key Laboratory of Medical Genomics, Ruijin Hospital Affiliated to Shanghai Jiao Tong University School of Medicine, Shanghai Institute of Hypertension, 197 Ruijin 2nd Road, Shanghai 200025, China

${ }^{2}$ Shanghai Key Laboratory of Hypertension, Shanghai Institute of

Hypertension, Shanghai 200025, China

Full list of author information is available at the end of the article
} 
(Continued from previous page)

Conclusions: We conclude that irbesartan prevents ACE2 deficiency-mediated pathological hypertrophy and myocardial fibrosis in ACE2 mutant mice via activation of the PPARy signaling and suppression of the TGF $\beta-C T G F$ -ERK signaling, resulting in attenuation of myocardial injury. Drugs targeting ACE2 and PPARy represent potential candidates to prevent and treat myocardial injury and related cardiac disorders.

Keywords: Angiotensin-converting enzyme 2, Irbesartan, Peroxisome proliferator-activated receptor- $\gamma$, Connective tissue growth factor, Myocardial injury

\section{Background}

Through the actions of its main biological peptide, angiotensin (Ang) II, the renin-angiotensin system (RAS) has been implicated in myocardial remodeling and heart failure [1-3]. Myocardial remodeling is thought to be the result of a continuum of cardiac structural and functional alterations within cellular and extracellular phenotype, including cardiac myocyte hypertrophy and the extracellular matrix (ECM) remodeling, which trigger disruption of the normal myocardial structure and increased clinical risk of heart failure $[1,2,4]$. Ang II plays an essential role in myocardial fibrosis by enhancing activation of transforming growth factor- $\beta$ (TGF $\beta$ ) and connective tissue growth factor (CTGF), which may further facilitate the extracellular signal-regulated kinase $1 / 2$ (ERK1/2) signaling [1,5,6]. Angiotensin-converting enzyme 2 (ACE2) is a negative regulator of the RAS and has recently been implicated in pathological hypertrophy and heart failure $[2,7,8]$. We have previously demonstrated that ACE2 overexpression prevents Ang II-mediated cardiovascular remodeling associated with reduction of TGF $\beta 1$ [9-12]. However, the exact roles and mechanisms of the ACE2 involved in myocardial hypertrophy, fibrosis and cardiac injury are largely unknown.

The angiotensin type I (AT1) receptor blocker irbesartan has unique chemical properties that enable it to partially activate the peroxisome proliferator-activated receptor- $\gamma$ (PPAR $\gamma)$, which is a nuclear receptor controlling cardiovascular hypertrophy and dysfunction $[3,8,13]$. Transgenic mice with cardiomyocyte-specific expression of PPAR $\gamma$ were protected from lipopolysaccharideinduced cardiac dysfunction [14]. Moreover, PPAR- $\gamma$ has been reported to be a protective regulator in cardiac injury by preventing activation of the CTGF and ERK signaling $[15,16]$. Our previous studies have revealed that the level of PPAR- $\gamma$ was downregulated in hypertensionmediated cardiovascular remodeling and injury associated with reduction of ACE2 expression [3]. However, the relationship between ACE2 gene and the PPAR $\gamma$ signaling remains to be fully elucidated. In this work, we directly assessed the hypothesis that irbesartan would exert its protective effects on ACE2 deficiency-mediated myocardial fibrosis and cardiac injury by the modulation of the PPAR $\gamma$ signaling pathway.

\section{Methods}

\section{Experimental animals and protocols}

Ten-week-old male ACE2 knockout (ACE2KO; Ace2 ${ }^{-/ y}$ ) mice were backcrossed into a pure $\mathrm{C} 57 \mathrm{BL} / 6$ background and treated with irbesartan (Bristol-Myers Squibb Co., Princeton, NJ) in their drinking water (50 mg. $\left.\mathrm{kg}^{-1} \cdot \mathrm{d}^{-1}\right)$ for 14 days as described previously [9,17]. The wild-type (WT; Ace $2^{+/ y}$ ) mice were used as the normal controls. Plasma Ang II and Ang-(1-7) levels were measured by radio-immunoassay analysis as described previously $[12,17]$. All experiments were approved and performed in accordance with the Guide for the Care and Use of Laboratory Animals published by the US National Institutes of Health (NIH Publication No.85-23, revised 1996), Shanghai Jiao Tong University School of Medicine and the Animal Research Ethics Committee at the Canadian Council on Animal Care.

\section{Echocardiography and myocardial ultrastructure observation}

Transthoracic echocardiography was performed and analyzed with a Vevo 770 highresolution imaging system equipped with a $30-\mathrm{MHz}$ transducer (RMV-707B; VisualSonics) in a blinded manner as described previously [2,18]. For transmission electron microscope analysis, samples of mice left ventricle tissues were immediately cut into small pieces and immersed in $2.5 \%$ glutaraldehyde as described previously [3]. The myocardial ultrastructure of mice was observed on a HITACHI600 electron microscope (Hitachi, Japan).

\section{RNA extraction and real-time PCR gene array}

The cardiac mRNA expression of PPARs and fibrosisrelated genes in WT and ACE2KO mice were examined using the real-time PCR gene array (The $\mathrm{RT}^{2}$ Profiler $^{\mathrm{rm}}$ PCR Array Mouse; http://www.sabiosciences. com/rt_pcr_product/HTML/PAMM-038Z.html). The total RNA was extracted from flash-frozen heart tissue using TRIzol extraction protocol (Invitrogen, CA) and purified using a RNeasy ${ }^{\oplus}$ MinElute $^{\mathrm{Tm}}$ Cleanup Kit (Qiagen, Valencia, CA). Subsequently, total RNA was reverse transcribed using the SuperScript III Reverse Transcriptase (Invitrogen, CA) and complementary DNA was amplified by PCR using the $2 \mathrm{X}$ SuperArray PCR Master Mix 
(SuperArray Bioscience, Frederick, MD). The Real-time PCR Gene Array was then performed on each sample using The PAMM-038Z RT ${ }^{2}$ Profiler $^{\text {TM }}$ PCR Array, according to the Manufacturer's instructions. Data were analyzed using the $\Delta \Delta \mathrm{Ct}$ method and expressed as fold changes of the upregulation or downregulation.

\section{TaqMan real-time PCR analysis}

TaqMan Real-time reverse transcription PCR were used to evaluate the cardiac mRNA levels as described previously $[2,18,19]$. The primer and probe for atrial natriuretic factor (ANF), $\beta$-myosin heavy chain $(\beta-\mathrm{MHC})$, TGF $\beta 1$, and fibronectin (FN1) are listed in Table 1. 18S rRNA was used as an endogenous control. All samples were run in triplicates.

\section{Western blot analysis}

Western blotting analysis was used to measure protein levels of mice hearts as described previously $[11,20]$. The primary antibody against ERK1/2 (44/42 kD), phosphoERK1/2 (44/42 kD), PPAR $\gamma$ (53, 57 kD), PPAR $\alpha$ (55 kD), PPAR $\delta$ (52 kD), CTGF (38 kD), Collagen I (150 kD), Collagen III (70 kD), AT1 (41 kD) and $\alpha$-tubulin $(55 \mathrm{kD})$ were obtained from Cell Signaling Technology (Beverly, MA), Abgent Biotech Co. (San Diego, CA), Abcam Inc. (Cambridge, MA) and Santa Cruz Biotechnology (Santa Cruz, CA), respectively. Aim proteins were detected by enhanced chemiluminescence using X-O-Mat X-ray film.

\section{Picro-sirius red and Masson trichrome staining}

Picro-sirius red (PSR) and Masson trichrome staining were carried out as previously described [12,17]. Masson trichrome-stained sections were used for assessment of overall tissue architecture and interstitial fibrosis. PSR staining of heart sections were used to assess for myocardial interstitial fibrosis.

\begin{tabular}{|c|c|c|}
\hline Genes & Primer/Probe & Sequence (Probe: 5'-FAM- -TAMRA-3') \\
\hline \multirow[t]{3}{*}{ ANF } & Forward Primer & 5'-GGAGGAGAAGATGCCGGTAGA-3' \\
\hline & Reverse Primer & 5'-GCTTCCTCAGTCTGCTCACTCA-3' \\
\hline & Probe & 5'-TGAGGTCATGCCCCCGCAGG-3' \\
\hline \multirow[t]{3}{*}{$\beta-\mathrm{MHC}$} & Forward Primer & 5'-GTGCCAAGGGCCTGAATGAG-3' \\
\hline & Reverse Primer & 5'-GCAAAGGCTCCAGGTCTGA-3' \\
\hline & Probe & 5'-ATCTTGTGCTACCCAGCTCTAA-3' \\
\hline \multirow[t]{3}{*}{ TGF- $\beta 1$} & Forward Primer & 5'-CCTGCAAGACCATCGACATG-3' \\
\hline & Reverse Primer & 5'-ACAGGATCTGGCCACGGAT-3' \\
\hline & Probe & 5'-CTGGTGAAACGGAAGCGCATCGAA-3' \\
\hline
\end{tabular}

"Primer/probe mix for $18 \mathrm{~S}$ and Fibronectin (product \#: Mm01256742_m1) were obtained from Applied Biosystems Inc. ANF atrial natriuretic factor; $\beta-M H C ~ \beta-m y o s i n$ heavy chain; TGF- $\beta 1$ transforming growth factor- $\beta 1$.

\section{Statistical analysis}

Values are expressed as mean \pm SEM. All statistical analyses were performed with SPSS software (Version 11.5) either by Student's $t$ test for comparison between two groups or by ANOVA followed by the Student-Newman -Keuls test for multiple-comparison testing as appropriate. A value of $P<0.05$ was considered to indicate statistically significance.

\section{Results}

Treatment with irbesartan attenuated ACE2 deficiencymediated myocardial fibrosis and ultrastructure injury in ACE2KO mice

We firstly evaluated the effects of ACE2 deficiency on myocardial fibrosis and injury. As shown in Figures 1 and 2, loss of ACE2 resulted in increased myocardial fibrosis and severe myocardial ultrastructure injury in the ACE2KO mice as assessed by PSR and Masson trichrome staining and transmission electron microscope analysis. Compared with Ace $2^{+/ y}$ mice, the myocardial collagen volume fraction (CVF) showed a marked increase in ACE2KO mice, which were significantly prevented by treatment with the AT1 blocker irbesartan (Figure 1) $(\mathrm{n}=4 ; P<0.05$ or $P<0.01$, respectively). In the ACE2KO hearts, myocardial myofilaments were disrupted and disarranged associated with vacuolar degenerational and swollen mitochondria (Figure 2). Notably, in response to irbesartan treatment, myocardial fibrosis and ultrastructure injury were alleviated in ACE2-null mice (Figures 1 \& 2).

Treatment with irbesartan prevented ACE2 deficiencymediated myocardial hypertrophy in ACE2KO mice with activation of the PPARY signaling

We next evaluated the regulatory roles of ACE2 deficiency on cardiac PPARs signaling and myocardial hypertrophy. As shown in Figure 3 and Table 2, loss of ACE2 resulted in marked decreases in cardiac expression of PPAR $\alpha$ and PPAR $\gamma$ in ACE2KO mice ( $\mathrm{n}=3-5$; $P<0.05$ or $P<0.01$, respectively), without affecting the expression of PPAR $\delta$. In addition, loss of ACE2 led to marked increases in left ventricular (LV) posterior wall thickness (LVPWT), LV weight (LVW) and the ratio of LVW and body weight (BW) in ACE2KO mice (Table 3) ( $\mathrm{n}=6-8 ; P<0.05$, respectively). TaqMan real-time PCR analysis revealed that there was an obvious increase in mRNA expression of hypertrophy-associated gene ANF (Figure 4A) in ACE2-deficient hearts $(n=6-8 ; P<0.01)$. Echocardiographic data revealed normal systolic function with no change in LV fractional shortening and ejection fraction between WT and ACE2KO mice (Table 3). Treatment with irbesartan largely prevented ACE2 deficiency-mediated increases in LVPWT, LVW, LVW/BW ratio (Table 3) and ANF mRNA level 

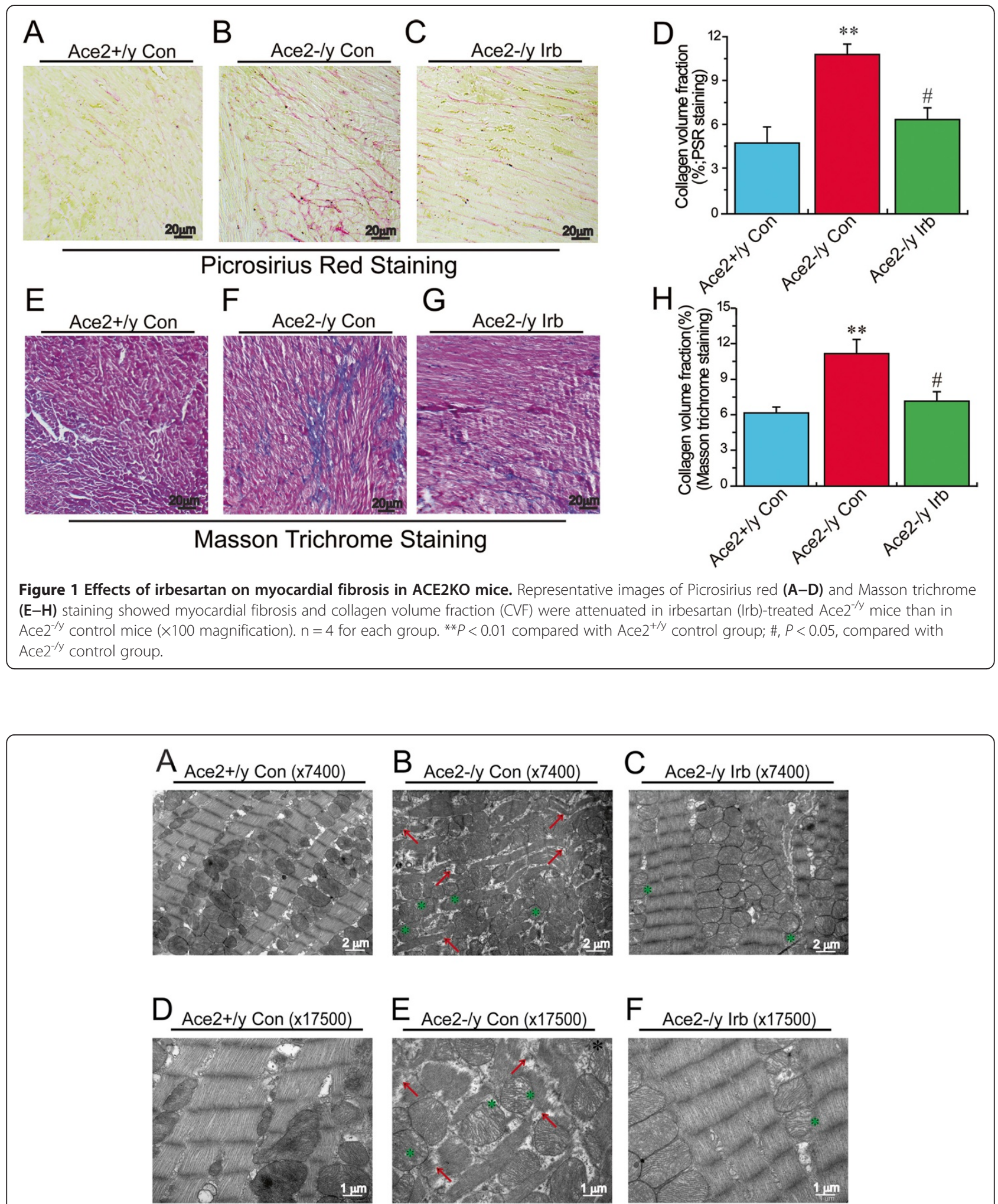

Figure 2 Effects of irbesartan on myocardial ultrastructure injury in ACE2KO mice. The myocardial ultrastructural changes were observed in $\mathrm{Ace}^{+/ y}$ control $(\mathbf{A}, \mathbf{D}), \mathbf{A c e}^{-/ y}$ control $(\mathbf{B}, \mathbf{E})$ and irbesartan (Irb)-treated Ace2 $2^{-1 / y}$ mice $\mathbf{( C , F )}$ by transmission electron microscope analysis $(\times 7400$ and $\times 17500$ magnification). Compared with $A c e 2^{+/ y}$ mice, severe myocardial ultrastructure injury was observed in Ace ${ }^{-/ y}$ mice, characterized with disruption or dissolution of myocardial myofilaments, myofilaments arranged irregularly and loosely (red arrow), and vacuolar degenerational and swollen mitochondria (green star). 

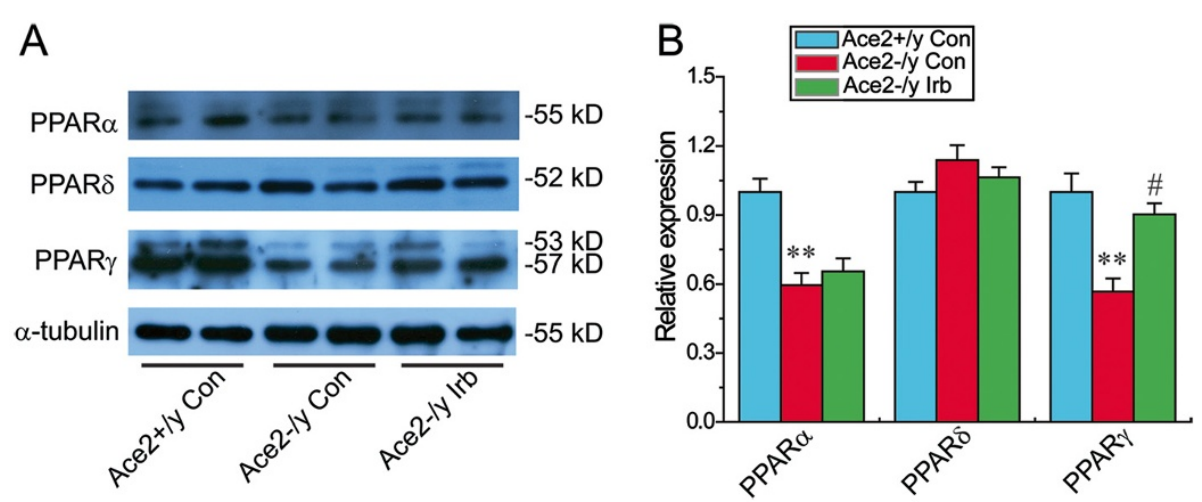

Figure 3 Cardiac protein levels of PPARa, PPARס and PPARy in mice. Representative Western blot (A) exhibited cardiac protein expression (B) of PPARa, PPARS, and PPARy in mice. a-tubulin was used as endogenous control. Irb = irbesartan, PPAR, peroxisome proliferator-activated receptor. $\mathrm{n}=5$ for each group. ${ }^{* *} P<0.01$ compared with Ace ${ }^{+/ y}$ control group; $\#, P<0.05$ compared with Ace2 ${ }^{-1 / y}$ control group.

(Figure 4A) in ACE2-null mice $(\mathrm{n}=6-8 ; P<0.05$, respectively). However, there was no change in cardiac $\beta$-MHC mRNA level among groups (Figure 4B). Intriguingly, the AT1 blocker irbesartan significantly upregulated cardiac mRNA (Table 4) and protein (Figure 3B) levels of PPARY in ACE2KO mice $(n=3-5$; $P<0.05$ or $P<0.01$, respectively). However, irbesartan did not increase the expression of PPAR $\alpha$ and PPAR $\delta$ (Figure 3 \& Table 4) in ACE2-deficient hearts $(n=3-5$; $P>0.05$, respectively). Our findings indicated the protective effects of irbesartan on myocardial hypertrophy in ACE2KO mice via activation of the PPARY signaling pathway.

\section{Treatment with irbesartan reversed ACE2 deficiency-} mediated activation of the TGF $\beta$-CTGF-ERK signaling in ACE2KO mice with decreased collagen level

We finally examined the effects of ACE2 deficiency on myocardial TGF $\beta-C T G F-E R K$ signaling and collagen production by real-time PCR gene array and Western blotting analysis. Compared with WT mice, the levels of TGF $\beta 1$, CTGF and collagen III genes were upregulated 2.62-, 4.17- and 2.37-fold in ACE2-deficient hearts (Table 2) (n=3; $P<0.05$ or $P<0.01$, respectively). After irbesartan treatment, the cardiac expression of TGF $\beta 1$, CTGF and collagen III were downregulated 2.55-, 3.03and 2.05-fold (Table 4) in ACE2KO mice $(\mathrm{n}=3 ; P<0.05$, respectively). Increased levels of TGF $\beta 1$, CTGF and collagen III were confirmed by TaqMan real-time PCR or Western blotting analysis, which showed increases in the mRNA or protein expression of TGF $\beta 1$, CTGF and collagen III in ACE2-deficient hearts along with augmented collagen I level (Figures 4 \& 5$)(\mathrm{n}=5-8 ; \quad P<0.01$, respectively). These changes were linked with enhanced phosphorylated level of ERK1/2 (Figure 6A) in ACE2deficient mice $(\mathrm{n}=5 ; P<0.01)$. More importantly, treatment with irbesartan significantly prevented ACE2 deficiency-induced increases in expression of TGF $\beta 1$ (Figure 4C), CTGF (Figure 5B), collagen I (Figure 5C) and collagen III (Figure 5D) and phosphorylated level of ERK1/2 (Figure 6A) in ACE2KO hearts $(n=5-8 ; P<0.05$ or $P<0.01$, respectively). However, there were no changes in cardiac mRNA levels of TGF $\beta 2$ and FN1 among groups (Figure 4, Tables $2 \& 3$ ). These changes were associated with a marked decrease in the levels of AT1 receptor and increases in plasma Ang-(1-7) level and the Ang-(1-7)/Ang II ratio in the irbesartan-treated ACE2KO mice (Figure 6) $(\mathrm{n}=5-8 ; P<0.05$ or $P<0.01$,

Table 2 The mRNA expression of PPAR and fibrosis-related genes in the ACE2-deficient hearts $(\mathbf{n}=\mathbf{3})$

\begin{tabular}{llll}
\hline Gene name & Description & Folds up- or down-regulation (Vs. WT mice) & $\boldsymbol{P}$ value \\
\hline PPARa & peroxisome proliferator activated receptor- $\alpha$ & -4.16 & 0.028 \\
PPAR & peroxisome proliferator activated receptor- $\delta$ & 1.02 & 0.968 \\
PPARY & peroxisome proliferator activated receptor- $\gamma$ & -2.06 & 0.004 \\
CTGF & connective tissue growth factor & 4.17 & 0.010 \\
Col3A1 & collagen, type III, alpha 1 & 2.37 & 0.021 \\
FN1 & fibronectin & 1.18 & 0.635 \\
TGF- $\beta 1$ & transforming growth factor- $\beta 1$ & 2.62 & 0.001 \\
TGF- $\beta 2$ & transforming growth factor- $\beta 2$ & 1.21 & 0.542 \\
\hline
\end{tabular}


Table 3 The general data in mice

\begin{tabular}{llll}
\hline & Ace2 $^{+/ y}$ control & Ace2 $^{-/ y}$ control & Ace2 $^{-/ y}$ Irbesartan \\
\hline $\mathbf{n}$ & $\mathbf{6}$ & $\mathbf{8}$ & $\mathbf{6}$ \\
HR (bpm) & $496 \pm 11$ & $494 \pm 12$ & $501 \pm 10$ \\
BW (g) & $26.53 \pm 0.72$ & $25.81 \pm 0.61$ & $26.68 \pm 0.52$ \\
LVW (mg) & $9.95 \pm 0.38$ & $12.18 \pm 0.31^{*}$ & $10.35 \pm 0.31$ \\
LVW/BW (mg/g) & $0.37 \pm 0.02$ & $0.47 \pm 0.03^{*}$ & $0.39 \pm 0.02$ \\
LVEDD (mm) & $3.83 \pm 0.03$ & $3.85 \pm 0.03$ & $3.86 \pm 0.04$ \\
LVESD (mm) & $1.75 \pm 0.04$ & $1.81 \pm 0.04$ & $1.79 \pm 0.05$ \\
LVFS (\%) & $54.32 \pm 0.93$ & $53.05 \pm 1.03$ & $53.61 \pm 1.14$ \\
LVEF (\%) & $62.03 \pm 3.01$ & $59.56 \pm 2.37$ & $63.83 \pm 3.89$ \\
LVPWT (mm) & $0.61 \pm 0.03$ & $0.78 \pm 0.03^{*}$ & $0.65 \pm 0.04$
\end{tabular}

HR heart rate; $B W$ body weight; $L V W$ left ventricular (LV) weight; LVEDD LV end diastolic diameter; LVESD LV end systolic diameter; LVFS LV fractional shortening; LVEF LV ejection fraction; LVPWT LV posterior wall thickness. $n=6-8$. Results are presented as mean \pm SEM. ${ }^{*}, p<0.05$ compared with all other groups.

\section{A}

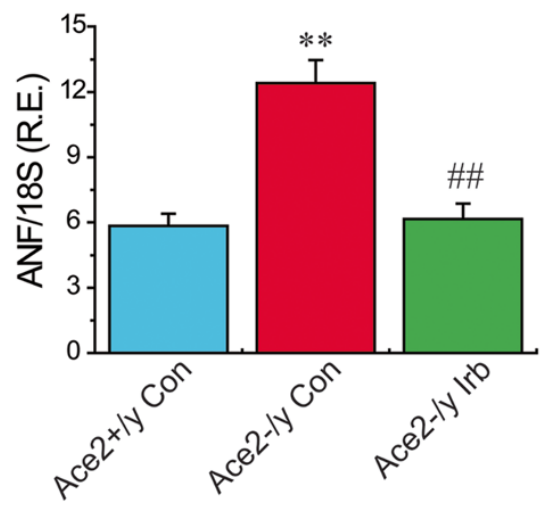

C

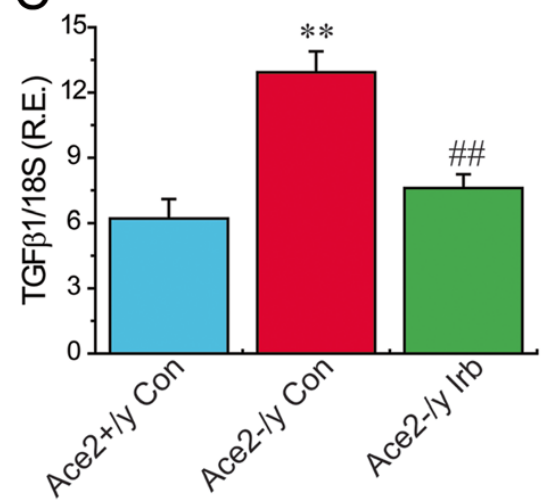

B

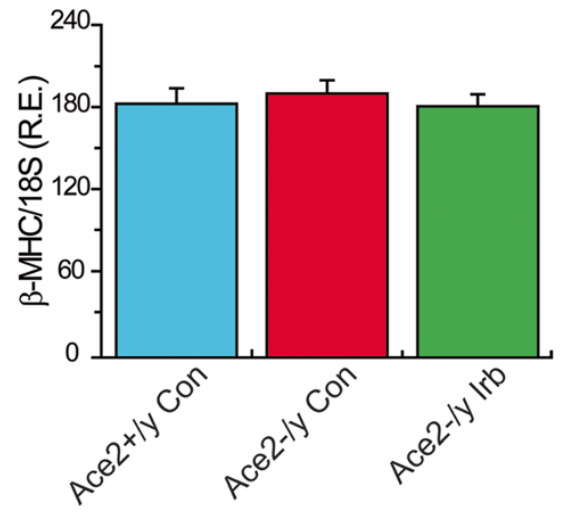

D

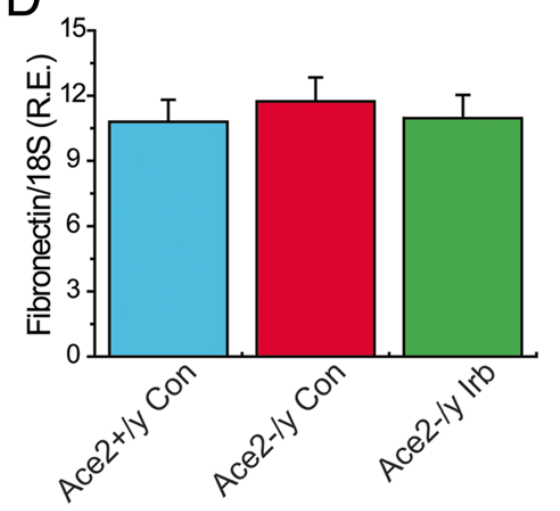

Figure 4 Cardiac mRNA levels of hypertrophy and fibrosis related genes in mice. TaqMan real-time PCR analysis revealed mRNA expression of hypertrophy and fibrosis related genes including ANF (A), $\beta-M H C$ (B), TGF $\beta 1$ (C), and fibronectin (D) in the hearts of mice. 185 rRNA was used as an endogenous control. R.E. = relative expression; ANF, atrial natriuretic factor; $\beta-M H C, \beta$-myosin heavy chain; TGF $\beta 1$, transforming growth

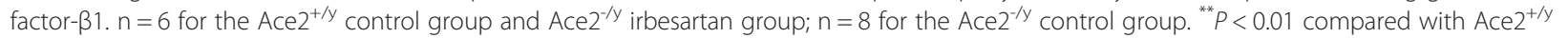
control group; \#\#, $P<0.01$ compared with $A c e 2^{-1 / y}$ control group. 
Table 4 The mRNA expression of PPAR and fibrosis-related genes in ACE2 deficient hearts after irbesartan treatment $(\mathbf{n}=3)$

\begin{tabular}{llll}
\hline Gene name & Description & Folds up- or down-regulation (Vs. ACE2KO mice) & $P$ value \\
\hline PPARa & peroxisome proliferator activated receptor-a & 1.46 & 0.655 \\
PPAR $\delta$ & peroxisome proliferator activated receptor- $\delta$ & -1.04 & 0.456 \\
PPARY & peroxisome proliferator activated receptor- $\gamma$ & 3.26 & 0.010 \\
CTGF & connective tissue growth factor & -3.03 & 0.019 \\
Col3A1 & collagen, type III, alpha 1 & -2.05 & 0.013 \\
FN1 & fibronectin & -1.55 & 0.107 \\
TGF- $\beta 1$ & transforming growth factor- $\beta 1$ & -2.55 & 0.008 \\
TGF- $\beta 2$ & transforming growth factor- $\beta 2$ & -1.24 & 0.789 \\
\hline
\end{tabular}

respectively). These observations confirmed detrimental effects of ACE2 deficiency on myocardial fibrosis and ultrastructure injury along with reduced Ang-(1-7) level and cardiac protective effects of irbesartan through normalization of AT1 receptor and the Ang-(1-7)/Ang II ratio in ACE2-null mice.

\section{Discussion}

In the present study, we provide the first evidence for cardiac protective roles of irbesartan in the ACE2 deficiency-mediated pathological hypertrophy, myocardial fibrosis and cardiac injury via activation of the
PPARy signaling pathway. We revealed that the deletion of the ACE2 gene resulted in pathological hypertrophy with marked increases in LVPWT, LVW and LVW/BW ratio and elevated ANF mRNA in ACE2-deficient hearts, without affecting the expression of $\beta$-MHC. These changes were linked with higher AT1 receptor level and lower Ang-(1-7) level in ACE2KO mice, which were significantly reversed by treatment with irbesartan. In addition, loss of ACE2 triggered myocardial ultrastructure injury, characterized with disruption or dissolution of myocardial myofilaments and mitochondrial vacuolar degeneration. However, there was no significant difference in
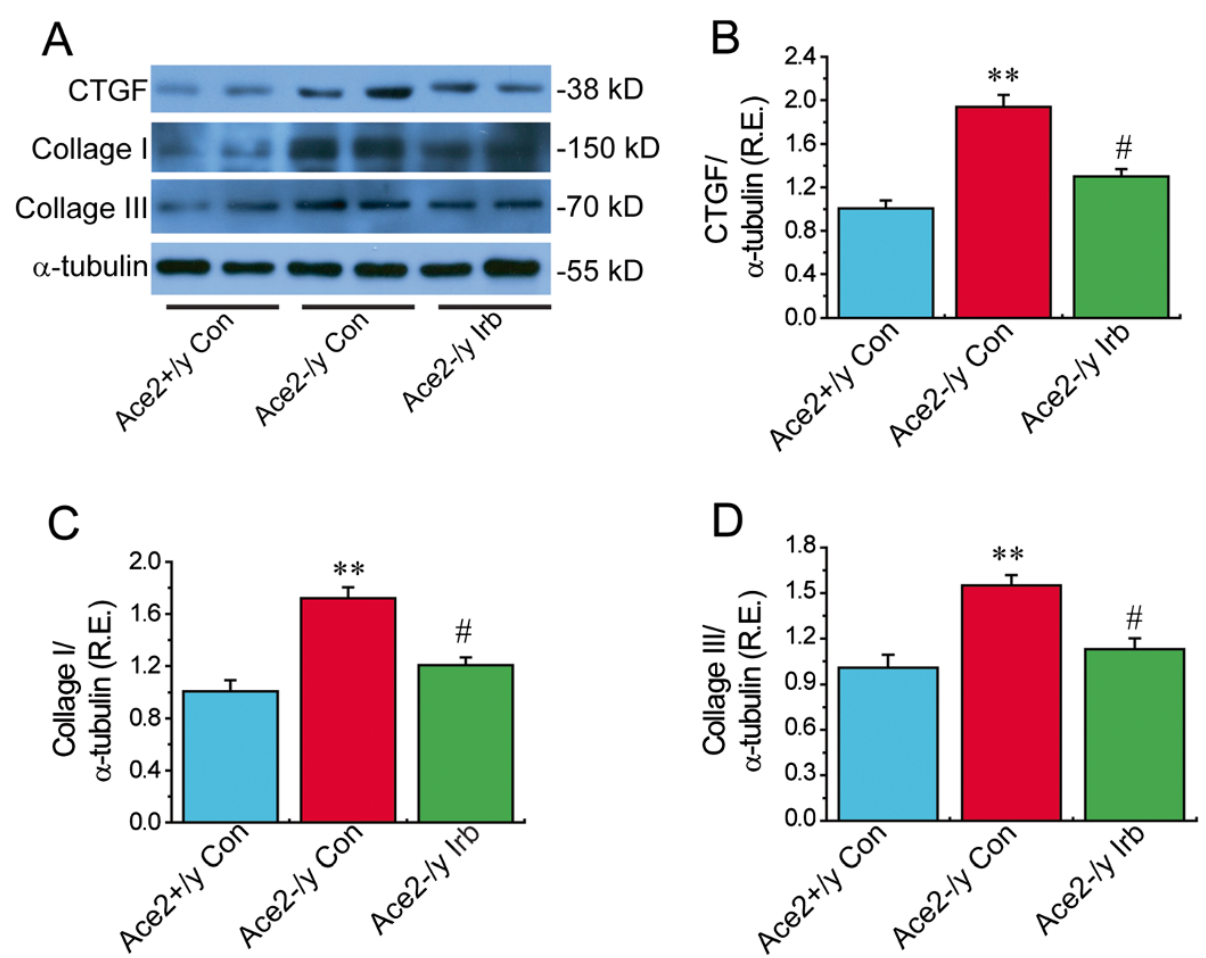

Figure 5 Cardiac protein levels of CTGF and collagen in mice. Representative Western blot (A) exhibited cardiac protein levels of CTGF (B), collagen I (C) and collagen III (D) in mice. a-tubulin was used as endogenous control. R.E. = relative expression; CTGF, connective tissue growth factor; Irb, irbesartan. $n=5$ for each group. ${ }^{* *} P<0.01$ compared with Ace ${ }^{+/ y}$ control group; \#, $P<0.05$ compared with Ace ${ }^{-/ y}$ control group. 


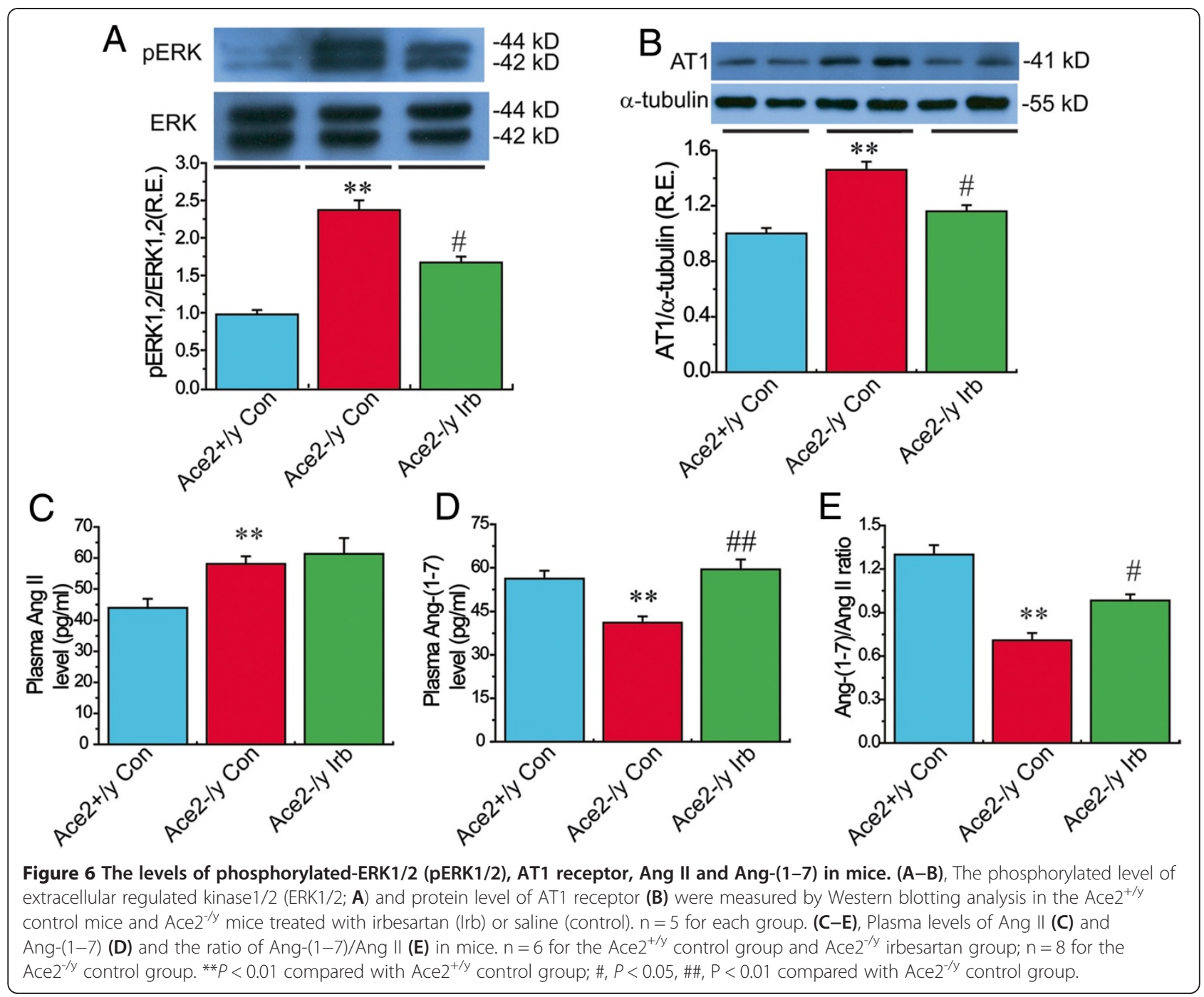

systolic function between WT and ACE2KO mice on the basis of the LVEF and LVFS by echocardiography analysis. It has become clear that PPARs exist in adipose and cardiovascular tissues as ligand-activated transcription factors and pharmacological activation of PPARs mediates strong organ-protective effects, including anti-hypertrophic and anti-fibrosis effects [3,13,21]. Recent studies have showed the cross-talk action between the RAS and PPARY in cardiovascular hypertrophy and remodeling [3,22,23]. Local RAS exists in the cardiovascular tissues, and ACE2, a negative regulator of the RAS, is present in various cell-types including the cardiofibroblasts, cardiomyocytes and coronary microcirculation $[20,24,25]$. ACE2 plays a critical role in the control of cardiac physiology and its altered expression is linked to major pathophysiological changes of the cardiovascular system $[24,26]$. To investigate the mechanism of pathological hypertrophy and myocardial injury in ACE2dificient status, we examined the changes in cardiac PPARs signaling. In ACE2KO hearts, lack of ACE2 results in marked decreases in expression of PPAR $\alpha$ and PPAR $\gamma$, without affecting the expression of PPAR $\delta$. This is in agreement with other reports showing that cardiac PPAR $\gamma$ mRNA was reduced in ACE2-null mice associated with myocardial hypertrophy [8]. The PPAR $\gamma$ deficiency has been exhibited to develop cardiac hypertrophy in PPAR $\gamma$ null mice [23]. More importantly, treatment with irbesartan remarkably attenuated ACE2-dificiency induced myocardial hypertrophy and ultrastructure injury along with augmentation of cardiac PPAR $\gamma$ level, without having a differential effect on the expression of PPAR $\alpha$ and PPAR $\delta$. Therefore, we speculate that upregulation of the PPAR $\gamma$ signaling is, at least in part, responsible for cardio-protective effects of irbesartan in ACE2-deficient mice. A limitation of the current study was that we did not examine the influence of the PPAR $\gamma$ antagonist GW9662 on actions of irbesartan. Treatment with GW9662 and irbesartan simultaneously 
would help to strengthen the mechanistic link between the PPAR signaling and irbesartan-mediated effects on cardiac remodeling and myocardial injury in ACE2-deficient mice.

The AT1 receptor blocker irbesartan has unique properties that enable it to partially activate the PPARy signaling, which has been implicated in controlling cardiovascular hypertrophy and fibrosis via suppression of the TGF $\beta-C T G F$ and ERK signaling pathways $[15,16,22]$. Evidence is emerging that the TGF $\beta$-CTGF-ERK signaling pathway regulates diverse cellular processes as ECM deposition and differentiation, as well as cell survival and proliferation, leading to cardiac fibrosis and structural injury $[15,22,27,28]$. TGF $\beta 1$ and CTGF are important mediators of the profibrotic effects of the RAS, such as the differentiation and proliferation of fibroblasts and collagen deposition, as Ang II increased the TGF $\beta$-CTGF-ERK signaling in various cells including cultured cardiomyocytes and animal models $[1,2,5,6,15,28]$. In contrast, PPAR $\gamma$ is a well-known antifibrotic factor in the cardiovascular system $[8,13,23,29]$. PPAR $\gamma$ mutant mice developed significantly more severe cardiac fibrosis to Ang II that correlated with increased expression of profibrotic genes collagen I and collagen III [23]. The PPAR $\gamma$ antagonist GW9662 prevented the cardiac protective effects of irbesartan in mice with myocardial hypertrophy and interstitial fibrosis [13]. Activation of PPAR- $\gamma$ has been reported to suppress cardiac expression of CTGF [16] and the phosphorylation signaling of ERK [15], thereby exerting protective effect on cardiac injury. In this study, we found that consistent with the induction of cardiac hypertrophy, loss of ACE2 led to enhanced CVF, severe myocardial fibrosis and adverse myocardial ultrastructure injury. Compared with WT mice, cardiac levels of TGF 31 , CTGF, collagen I and collagen III were higher in ACE2KO mice associated with lower PPARy level and higher phosphorylated ERK1/2 level. This is in agreement with other reports showing that the lower expression of PPAR $\gamma$ was observed in ACE2-deficient hearts associated with higher mRNA levels of TGF $\beta 1$, collagen I and collagen III and cardiovascular fibrosis [8]. However, there was no change in cardiac TGF $\beta 2$ and fibronectin mRNA levels between ACE2KO and WT mice. More importantly, administration of irbesartan significantly reversed ACE2 deficiency-induced increases in CVF, TGF $\beta 1$, CTGF, collagen I, collagen III and phosphorylated ERK1/2 and remarkably attenuated myocardial fibrosis and ultrastructure injury, likely driven by the elevation in PPAR $\gamma$ levels in ACE2KO mice. Taken together, our data demonstrate that treatment with irbesartan reversed ACE2 deficiency-mediated cardiac fibrosis and adverse myocardial injury via activation of the PPARy signaling and suppression of the TGF $\beta$-CTGF-ERK signaling.

\section{Conclusions}

In summary, loss of ACE2 enhances the susceptibility to pathological hypertrophy and myocardial fibrosis, resulting in adverse myocardial ultrastructure deterioration. In contrast, treatment with irbesartan prevents ACE2 deficiencymediated cardiac hypertrophy and myocardial ultrastructure injury in ACE2 mutant mice with elevation of the PPAR $\gamma$ level and suppression of the TGF $\beta-C T G F-E R K$ signaling. These results indicate cardiac protective roles of irbesartan in the prevention of myocardial hypertrophy, fibrosis and cardiac injury via activation of the PPAR $\gamma$ signaling and support the notion that irbesartan functions as a partial agonist of PPAR $\gamma$. Targeting ACE2 and PPAR $\gamma$ has potential therapeutic importance for modulating cardiac remodeling, fibrosis and myocardial injury. Future studies are required to more precisely clarify regulatory roles of ACE2 and PPAR $\gamma$ as biomarkers of myocardial injury and related heart diseases.

\section{Abbreviations}

ACE2: Angiotensin-converting enzyme 2; ANF: Atrial natriuretic factor; Ang II: Angiotensin II; $\beta$-MHC: $\beta$-myosin heavy chain; BW: Body weight; CTGF: Connective tissue growth factor; ECM: Extracellular matrix;

ERK1/2: Extracellular signal-regulated kinase 1/2; HR: Heart rate; KO: Knockout; LV: Left ventricular; LVPWT: Left ventricular posterior wall thickness; LWW: Left ventricular weight; LVEDD: Left ventricular end diastolic diameter; LVESD: Left ventricular end systolic diameter; LVFS: Left ventricular fractional shortening; LVEF: Left ventricular ejection fraction; PPARY: Peroxisome proliferatoractivated receptor- $\gamma$; PSR: Picro-sirius red; RAS: Renin-angiotensin system; TGF $\beta 1$ : Transforming growth factor- $\beta 1$.

\section{Competing interests}

The authors declare that they have no competing interests.

\section{Authors' contributions}

$J C Z$, QHS, PJG and GYO conceived the study design, participated in the acquisition of data and drafted the manuscript. ZZZ, HYJ, BS and YLX carried out the experiments and participated in the acquisition of data, analysis and interpretation. TZ and JMP have been involved in analyzing the data and provided critical advice. LL and DLZ performed the statistical analysis and helped to draft the manuscript. All authors read and approved the final manuscript.

\section{Acknowledgements}

This work was supported by the National Natural Science Foundation of China (81370362, 81170246, 30973522, 81230071 \& 81270801), the Shanghai Pujiang Talents Program of Shanghai Science and Technology Committee (11PJ1408300), and the Canadian Institute for Health Research (86602). Dr. Zhong is one of SMC Morningstar Distinguished Young Scholars of Shanghai Jiao Tong University in China and a Fellow of Alberta Innovates-Health

Solution in Canada. We gratefully acknowledge the technical assistance from the University of Alberta and Shanghai Jiao Tong University.

\section{Author details}

'State Key Laboratory of Medical Genomics, Ruijin Hospital Affiliated to Shanghai Jiao Tong University School of Medicine, Shanghai Institute of Hypertension, 197 Ruijin 2nd Road, Shanghai 200025, China. ${ }^{2}$ Shanghai Key Laboratory of Hypertension, Shanghai Institute of Hypertension, Shanghai 200025, China. ${ }^{3}$ Department of Cardiology and Institute of Clinical Medicine Research, Affiliated Hospital of Zunyi Medical College, Zunyi 563003, China. ${ }^{4}$ Department of Mental Health, Ruijin Hospital, School of Medicine, Shanghai Jiao Tong University, Shanghai 200025, China. ${ }^{5}$ Divsion of Cardiology, Department of Medicine, University of Alberta, Mazankowski Alberta Heart Institute, Edmonton T6G 2S2, Canada. ${ }^{6}$ Department of Cardiology, Ruijin Hospital, School of Medicine, Shanghai Jiao Tong University, Shanghai 200025, China. 'Department of Pediatrics, Ruijin Hospital, School of Medicine, Shanghai Jiao Tong University, Shanghai 200025, China. Institute of Molecular Biotechnology of the Austrian Academy of Sciences, Vienna, Austria. 
Received: 25 July 2013 Accepted: 24 September 2013

Published: 25 September 2013

\section{References}

1. Wang M, Zhang J, Walker SJ, Dworakowski R, Lakatta EG, Shah AM: Involvement of NADPH oxidase in age-associated cardiac remodeling. J Mol Cell Cardiol 2010, 48:765-772.

2. Zhong J, Basu R, Guo D, Chow FL, Byrns S, Schuster M, Loibner H, Wang XH, Penninger JM, Kassiri Z, Oudit GY: Angiotensin-converting enzyme 2 suppresses pathological hypertrophy, myocardial fibrosis, and cardiac dysfunction. Circulation 2010, 122:717-728.

3. Zhong JC, Ye JY, Jin HY, Yu X, Yu HM, Zhu DL, Gao PJ, Huang DY, Shuster M, Loibner H, Guo JM, Yu XY, Xiao BX, Gong ZH, Penninger JM, Oudit GY: Telmisartan attenuates aortic hypertrophy in hypertensive rats by the modulation of ACE2 and profilin-1 expression. Regul Pept 2011, 166:90-97.

4. Zhang HF, Xie SL, Chen YX, Mai JT, Wang JF, Zhu WL, Zhu LG: Altered serum levels of IL-33 in patients with advanced systolic chronic heart failure: correlation with oxidative stress. J Transl Med 2012, 10:120.

5. Ebrahimian T, Li MW, Lemarié CA, Simeone SM, Pagano PJ, Gaestel M, Paradis $P$, Wassmann S, Schiffrin EL: Mitogen-activated protein kinaseactivated protein kinase 2 in angiotensin II-induced inflammation and hypertension: regulation of oxidative stress. Hypertension 2011, 57:245-254.

6. Wu SH, Lu C, Dong L, Chen ZQ: Signal transduction involved in CTGFinduced production of chemokines in mesangial cells. Growth Factors 2008, 26:192-200.

7. Iwai M, Nakaoka H, Senba I, Kanno H, Moritani T, Horiuchi M: Possible involvement of angiotensin-converting enzyme 2 and Mas activation in inhibitory effects of angiotensin II Type 1 receptor blockade on vascular remodeling. Hypertension 2012, 60:137-144.

8. Moritani T, Iwai M, Kanno H, Nakaoka H, Iwanami J, Higaki T, Ishii E, Horiuchi M: ACE2 deficiency induced perivascular fibrosis and cardiac hypertrophy during postnatal development in mice. J Am Soc Hypert 2013, 7:259-266.

9. Jin HY, Song B, Oudit GY, Davidge ST, Yu HM, Jiang YY, Gao PJ, Zhu DL, Ning G, Kassiri Z, Penninger JM, Zhong JC: ACE2 deficiency enhances angiotensin II-mediated aortic profilin-1 expression, inflammation and peroxynitrite production. PLoS One 2012, 7:e38502.

10. Patel VB, Bodiga S, Fan D, Das SK, Wang Z, Wang W, Basu R, Zhong J, Kassiri Z, Oudit GY: Cardioprotective effects mediated by angiotensin II type 1 receptor blockade and enhancing angiotensin 1-7 in experimental heart failure in angiotensin-converting enzyme 2-null mice. Hypertension 2012, 59:1195-1203.

11. Zhong JC, Huang DY, Yang YM, Li YF, Liu GF, Song XH, Du K: Upregulation of angiotensin-converting enzyme 2 by all-trans retinoic acid in spontaneously hypertensive rats. Hypertension 2004, 44:907-912.

12. Zhong J, Guo D, Chen CB, Wang W, Schuster M, Loibner H, Penninger JM, Scholey JW, Kassiri Z, Oudit GY: Prevention of angiotensin II-mediated renal oxidative stress, inflammation, and fibrosis by angiotensinconverting enzyme 2. Hypertension 2011, 57:314-322.

13. Kusunoki H, Taniyama Y, Rakugi H, Morishita R: Cardiac and renal protective effects of irbesartan via peroxisome proliferator-activated receptory-hepatocyte growth factor pathway independent of angiotensin II Type 1a receptor blockade in mouse model of saltsensitive hypertension. J Am Heart Assoc 2013, 2:e000103.

14. Drosatos K, Khan RS, Trent CM, Jiang H, Son NH, Blaner WS, Homma S, Schulze PC, Goldberg IJ: Peroxisome proliferator-activated receptor- $\gamma$ activation prevents sepsis-related cardiac dysfunction and mortality in mice. Circ Heart Fail 2013, 6:550-562.

15. Koh EJ, Yoon SJ, Lee SM: Losartan protects liver against ischaemia/ reperfusion injury through PPAR- $\gamma$ activation and receptor for advanced glycation end-products down-regulation. Br J Pharmacol 2013, 169:1404-1416,

16. Lee SW, Won JY, Kim WJ, Lee J, Kim KH, Youn SW, Kim JY, Lee EJ, Kim YJ, Kim KW, Kim HS: Snail as a potential target molecule in cardiac fibrosis: paracrine action of endothelial cells on fibroblasts through snail and CTGF axis. Mol Ther 2013. 10.1038/mt.2013.146. Epub ahead of print.

17. Kassiri Z, Zhong J, Guo D, Basu R, Wang X, Liu PP, Scholey JW, Penninger $J M$, Oudit GY: Loss of angiotensin-converting enzyme 2 accelerates maladaptive left ventricular remodeling in response to myocardial infarction. Circ Heart Fail 2009, 2:446-455.

18. Bodiga S, Zhong JC, Wang W, Basu R, Lo J, Liu GC, Guo D, Holland SM, Scholey JW, Penninger JM, Kassiri Z, Oudit GY: Enhanced susceptibility to biomechanical stress in ACE2 null mice is prevented by loss of the p47 (phox) NADPH oxidase subunit. Cardiovasc Res 2011, 91:151-161.

19. Cai M, Wu J, Mao C, Ren J, Li P, Li X, Zhong J, Xu C, Zhou T: A Lectin-EGF antibody promotes regulatory $T$ cells and attenuates nephrotoxic nephritis via DC-SIGN on dendritic cells. J TransI Med 2013, 11:103.

20. Song $B$, Jin $H, Y u X$, Zhang $Z, Y u H, Y e J, X u Y$, Zhou T, Oudit GY, Ye JY, Chen C, Gao P, Zhu D, Penninger JM, Zhong JC: Angiotensin-converting enzyme 2 attenuates oxidative stress and VSMC proliferation via the JAK2/STAT3/SOCS3 and profilin-1/MAPK signaling pathways. Regul Pept 2013, 185:44-51. 10.1016/j.regpep.2013. 06.007. Epub ahead of print.

21. Ares-Carrasco S, Picatoste B, Camafeita E, Carrasco-Navarro S, Zubiri I, Ortiz A, Egido J, López JA, Tuñón J, Lorenzo O: Proteome changes in the myocardium of experimental chronic diabetes and hypertension: role of PPARa in the associated hypertrophy. J Proteomics 2012, 75:1816-1829.

22. Asakawa M, Takano H, Nagai T, Uozumi H, Hasegawa H, Kubota N, Saito T, Masuda Y, Kadowaki T, Komuro I: Peroxisome proliferator-activated receptor gamma plays a critical role in inhibition of cardiac hypertrophy in vitro and in vivo. Circulation 2002, 105:1240-1246.

23. Kis A, Murdoch C, Zhang M, Siva A, Rodriguez-Cuenca S, Carobbio S, Lukasik A, Blount M, O'Rahilly S, Gray SL, Shah AM, Vidal-Puig A: Defective peroxisomal proliferators activated receptor gamma activity due to dominant-negative mutation synergizes with hypertension to accelerate cardiac fibrosis in mice. Eur J Heart Fail 2009, 11:533-541.

24. Hashimoto T, Perlot T, Rehman A, Trichereau J, Ishiguro H, Paolino M, Sigl V, Hanada T, Hanada R, Lipinski S, Wild B, Camargo SM, Singer D, Richter A, Kuba K, Fukamizu A, Schreiber S, Clevers H, Verrey F, Rosenstiel P, Penninger JM: ACE2 links amino acid malnutrition to microbial ecology and intestinal inflammation. Nature 2012, 487:477-481.

25. Zhong JC, Yu XY, Lin QX, Li XH, Huang XZ, Xiao DZ, Lin SG: Enhanced angiotensin converting enzyme 2 regulates the insulin/Akt signalling pathway by blockade of macrophage migration inhibitory factor expression. Br J Pharmacol 2008, 153:66-74.

26. Kuba K, Imai Y, Penninger JM: Multiple functions of angiotensinconverting enzyme 2 and its relevance in cardiovascular diseases. Circ J 2013, 77:301-308.

27. Ko WC, Hong CY, Hou SM, Lin CH, Ong ET, Lee CF, Tsai CT, Lai LP: Elevated expression of connective tissue growth factor in human atrial fibrillation and angiotensin II-treated cardiomyocytes. Circ J 2011, 75:1592-1600.

28. Leask A: Potential therapeutic targets for cardiac fibrosis: TGFbeta, angiotensin, endothelin, CCN2, and PDGF, partners in fibroblast activation. Circ Res 2010, 106:1675-1680.

29. Morrow JP, Katchman A, Son NH, Trent CM, Khan R, Shiomi T, Huang H, Amin V, Lader JM, Vasquez C, Morley GE, D'Armiento J, Homma S, Goldberg IJ, Marx SO: Mice with cardiac overexpression of peroxisome proliferatoractivated receptor $\gamma$ have impaired repolarization and spontaneous fatal ventricular arrhythmias. Circulation 2011, 124:2812-2821.

doi:10.1186/1479-5876-11-229

Cite this article as: Zhang et al:: Cardiac protective effects of irbesartan via the PPAR-gamma signaling pathway in angiotensin-converting enzyme 2-deficient mice. Journal of Translational Medicine 2013 11:229.

\section{Submit your next manuscript to BioMed Central and take full advantage of:}

- Convenient online submission

- Thorough peer review

- No space constraints or color figure charges

- Immediate publication on acceptance

- Inclusion in PubMed, CAS, Scopus and Google Scholar

- Research which is freely available for redistribution 